LEVELT, W. J. M., Note on the distribution of dominance times in binocular

rivalry, British Journal of Psychology, 58 (1967) p. 143

Bv. J. Psychol. (1967), 58, 1 and 2, pp. 143-145

Printed in Great Britain

\title{
NOTE ON THE DISTRIBUTION OF DOMINANCE TIMES IN BINOCULAR RIVALRY
}

\author{
BY W. J. M. LEVELT* \\ Institute for Perception RVO-TNO, Soesterberg, The Netherlands
}

\begin{abstract}
The dominance periods of a stimulus in binocular rivalry show a characteristic time distribution that gives important clues as to the underlying mechanism in alternation. It is shown that the distribution ean be approximated by a $\Gamma$-function, which turns out to have a positive integral exponent. With an integral exponent the function describes a Poisson distribution. This suggests the existence of an underlying dominance generating process that is discrete in nature. The parameters of this process are determined by properties of the recessive stimulus in the other eye. The disereto events may be 'flicks' of eye movement.
\end{abstract}

This note is an appendix to the writer's paper in the previous issue of this Journal (Levelt, 1966). The reader is referred to that paper for the experiments on which the present discussion is based and also for more precise definitions of the symbols used. The main conclusion of that article was that in binocular rivalry the mean duration of the predominance period of one eye is a function only of the stimulus strength in the other eye.

Further insight in how the shift in eye dominance is generated may be obtained by considering the distribution of dominance times $\left(t_{2}\right)$ of, say, the left eye during rivalry. This analysis is based on the alternation recordings of ten subjects for two different stimulus conditions. As a first step, means $\left(\bar{t}_{l}\right)$ and standard deviations $\left(s_{l}\right)$ were calculated over the sample of dominance times of each of the ten subjects under the two stimulus conditions. Relations between means and standard deviations were shown in Figs. 6 and 7 of Levelt (1966) and they can be summarized in both cases by the expression $\vec{t}_{l}=2 s_{l}$. This relation holds fairly well for both large and small $t_{l}$. It at once excludes the possibility that dominance times can be described by an exponential distribution of 'holding times', for, if the chance of no left-right shift during $t$ is $P_{\mathrm{o}}(t)=e^{-\lambda t}$, then the mean time $\vec{t}=1 / \lambda$, and also $\sigma=1 / \lambda$. This does not agree with the findings.

Because of the constant ratio, $\bar{t}_{l} / s_{l}$, the $t$-values for each subject in each of the two experiments were divided by the mean $(t)$ of these values in order to put all twenty distributions on the same seale. Hence all of the rescaled distributions have the same mean $\bar{t}=1$. From these values a single histogram of the $t_{l}$-distribution was made for both experiments and all subjects (see Fig. 1).

The distribution of the $t_{l}$-values can be approximated by a function of the form

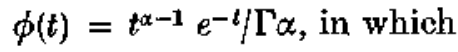

$$
\Gamma \alpha=\int_{0}^{\infty} t^{\alpha-1} e^{-t} d t
$$

(for positive integers $\Gamma \alpha=(\alpha-1)$ !). The $t$-distribution, $f(t)$, for each subject, and also the distribution of Fig. 1, may be conceived of as deformations of $\phi(t)$, with

* Now at the Department of Psychology, University of Groningen, The Netherlands. 
$f(t)=\lambda \phi(\lambda t)$ in which $\lambda$ represents a scale factor. The mean of the $t$-distribution is

the variance is

$$
\mu=\int_{0}^{\infty}\left\{t^{\alpha} e^{-t} / \Gamma \alpha\right\} d t=\alpha
$$

$$
\sigma^{2}=\int_{0}^{\infty}\left\{t^{\alpha+1} e^{-t} / \Gamma \alpha\right\} d t-\mu^{2}=\alpha,
$$

hence the standard deviation $\sigma=\sqrt{ } \alpha$.

The experimental requirement that $\mu=2 \sigma$ is met by $\alpha=4(\mu=4, \sigma=2)$. Hence the best fitting $\phi(t)=t^{3} e^{-t / 3}$ !, and

$$
f(t)=\lambda(\lambda t)^{3} e^{-\lambda t / 3 !}
$$

The mean of this distribution is $\bar{t}=4 / \lambda$, whereas its standard deviation is $2 / \lambda$. This function has been fitted to the histogram in Fig. 1. The area under the curve was

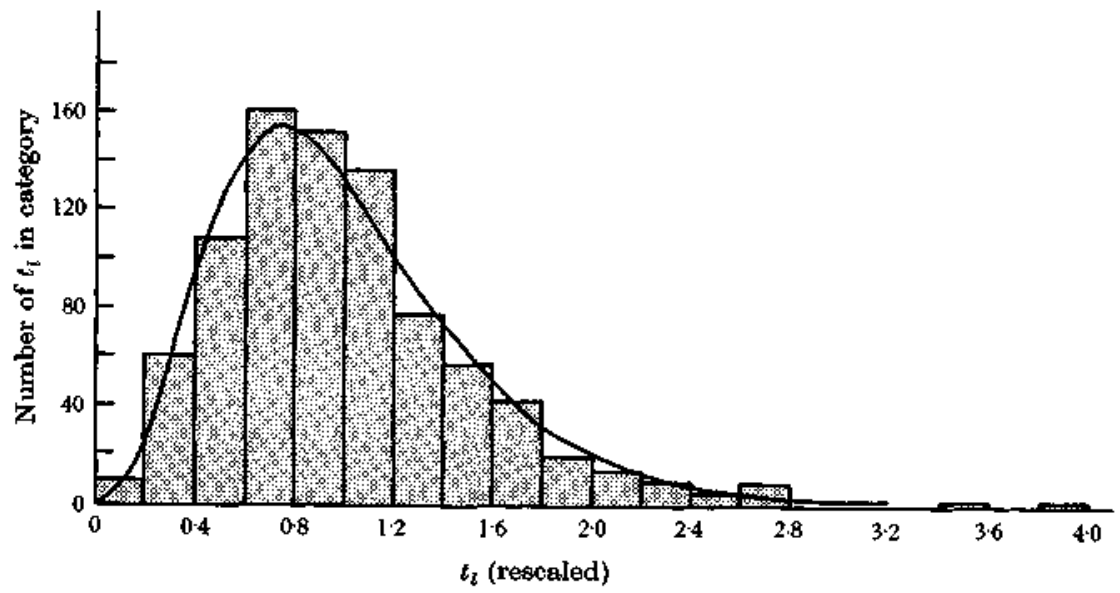

Fig. 1. Histogram of $t_{l}$-distribution $\left(\bar{t}_{l}=1\right)$, with best fitting density funetion $\phi(t)=t^{t} e^{-t} / 6$.

made equal to the area under the histogram, and a scale factor $\lambda=4$ had to be used in order to give $\bar{t}=1$, since the histogram had been rescaled to have a mean of unity. The approximation is fair, but it is realized that other functions may fit as well. The question of interest, however, is whether the function ean be understood as an expression of some underlying mechanism.

In the previous paper (Levelt, 1966) it was shown that $t_{l}$ is independent of what was there called $\lambda_{l}$, the strength of the left eye stimulus. It is not accidental that the same term $\lambda$ has been used above. The scale factor $\lambda$ in expression (1) may be taken to be the strength of the stimulus presented to the other eye, and evidence was indeed presented that $\bar{t}_{t}$ and the right eye stimulus strength, $\lambda_{r}$, were related by a monotonic decreasing function $\bar{t}_{t}=f\left(\lambda_{r}\right)$ (see Levelt, 1966, p. 227). It is now possible to specify this monotonic decreasing function as $\bar{t}_{l}=4 / \lambda_{r}$, in which an increase of $\lambda_{r}$ implies a reduction of $\bar{t}_{l}$.

One possible mechanism that could account for an effect of stimulus strength so that a function of form (1) results, is a summative one in which the recessive stimulus produces a series of randomly distributed 'exeitation spikes'. If the chance of no 
spike occurring in $t$ is $P_{o}(t)=e^{-\lambda t}$, the waiting time for one spike has a probability density function $f_{1}(t)=\lambda e^{-\lambda t}$. The waiting time up to the $n$th spike can be found by applying

$$
f_{n}(t)=\int_{-\infty}^{\infty} f_{n-1}(u) f_{1}(t-u) d u
$$

(on the assumption that the waiting time for the next spike is independent of the previous waiting times). For $n=4, f_{4}(t)=\lambda(\lambda t)^{3} e^{-\lambda t / 3}$ ! which is identical with expression (1), above.

This may be interpreted in the sense that the summative effect of four successive spikes from the recessive stimulus is necessary and sufficient to re-establish dominance for that stimulus. The symbol $\lambda$ now represents simply the number of spikes per unit time. The experiments gave values of $\bar{t}_{l}$ of about $2 \cdot 6 \mathrm{sec}$. Taking $\vec{t}_{l}=\mathbf{4} / \lambda_{r}=\mathbf{2 \cdot 6}$, gives $\lambda=1.54 \mathrm{sec}$ or an average interspike interval of about $0.65 \mathrm{sec}$. However, there are large individual differences in $\lambda_{r}$. This interspike time might be associated with 'flicks' in eye movement, suggesting some summating effect of time contours. It is not possible at present to give a more psychophysical account of such a summative process, and further elaboration of the term 'excitation spike' as used in this note would therefore be premature.

\section{REFERENCES}

Levelt, W. J. M. (1966). The alternation process in binoeular rivalry. Br. J. Psychol. 57, $225-38$.

(Manuscript received 19 May 1967) 